\title{
A New Approach to Prevention of Knee Osteoarthritis: Reducing Medial Load in the Contralateral Knee
}

\author{
RICHARD K. JONES, GRAHAM J. CHAPMAN, ANDREW H. FINDLOW, LAURA FORSYTHE, \\ MATTHEW J. PARKES, JAWAD SULTAN, and DAVID T. FELSON
}

ABSTRACT. Objective. Few if any prevention strategies are available for knee osteoarthritis (OA). In those with symptomatic medial OA, the contralateral knee may be at high risk of disease, and a reduction in medial loading in that knee might prevent disease or its progression there. Our aim was to determine how often persons with medial OA on 1 side had either concurrent or later medial OA on the contralateral side, and whether an intervention known to reduce medial loading in affected knees with medial OA might reduce medial loading in the contralateral knee. Lateral wedge insoles reduce loading across an affected medial knee but their effect on the contralateral knee is unknown.

Methods. To determine the proportion of persons with medial knee OA who had concurrent medial contralateral OA or developed contralateral medial OA later, we examined knee radiographs from the longitudinal Framingham Osteoarthritis Study. Then, to examine an approach to reducing medial load in the contralateral knee, 51 people from a separate study with painful medial tibiofemoral OA underwent gait analysis wearing bilateral controlled shoes with no insoles, and then with 2 types of wedge insoles laterally posted by $5^{\circ}$. Primary outcome was the external knee adduction moment (EKAM) in the contralateral knee. Nonparametric CI were constructed around the median differences in percentage change in the affected and contralateral sides.

Results. Of Framingham subjects with medial radiograph knee OA, 137/152 (90\%) either had concurrent contralateral medial OA or developed it within 10 years. Of those with medial symptomatic knee OA, 43/67 (64\%) had or developed the same disease state in the contralateral knee. Compared to a control shoe, medial loading was reduced substantially on both the affected (median percentage EKAM change $-4.84 \%$; $95 \% \mathrm{CI}-11.33 \%$ to $-0.65 \%$ ) and contralateral sides (median percentage EKAM change $-9.34 \%$; $95 \%$ CI $-10.57 \%$ to $-6.45 \%$ ).

Conclusion. In persons with medial OA, the contralateral knee is also at high risk of medial OA. Bilateral reduction in medial loading in knees by use of strategies such as lateral wedge insoles might not only reduce medial load in affected knees but prevent knee OA or its progression on the contralateral side. (First Release Jan 15 2013; J Rheumatol 2013;40:309-15; doi:10.3899/jrheum.120589)

Key Indexing Terms: OSTEOARTHRITIS

KNEE

Knee osteoarthritis (OA) is a chronic and widely prevalent disease that affects about $13 \%$ of individuals aged 65 years and older ${ }^{1}$. Knee OA most often presents in the medial compartment of the joint, with a prevalence 5-10 times

From the University of Salford, School of Health Sciences, Salford; and Arthritis Research UK Epidemiology Unit, Manchester Academic Health Science Centre, The University of Manchester, Manchester, UK.

Supported by a program grant from Arthritis Research UK (grant 18676) and by US National Institutes of Health (grant AR47785).

R.K. Jones, PhD, University of Salford, School of Health Sciences, Arthritis Research UK Epidemiology Unit, Manchester Academic Health Science Centre, The University of Manchester; G.J. Chapman, PhD; A.H. Findlow, PhD, University of Salford, School of Health Sciences; L. Forsythe, BSc; M.J. Parkes, BSc; D.T. Felson, MD, Arthritis Research UK Epidemiology Unit, Manchester Academic Health Science Centre, The University of Manchester; J. Sultan, MBBS, MRCS (Ed), MSc, University Hospital of South Manchester, Wythenshawe Hospital, Department of Orthopaedics, Manchester.

Address correspondence to Dr. R.K. Jones, Directorate of Prosthetics and Orthotics and Podiatry, School of Health Sciences, Brian Blatchford

Building, University of Salford, Salford, M6 6PU, UK.

E-mail:r.k.jones@salford.ac.uk

Full Release Article. For details see Reprints/Permissions at jrheum.org Accepted for publication November 21, 2012.

\section{PREVENTION}

KINETICS

higher than disease in the lateral compartment in Western populations ${ }^{2,3}$. One suggested reason for this is that about $60 \%$ of load goes through the medial side of the knee during walking 4 .

No strategies have been clearly shown to prevent OA or its progression. Among those advocated are weight loss and exercise. For both, evidence is limited and both are difficult for patients to adhere to in the long term. Another strategy would be to focus on those with painful OA in 1 knee who already have or are at high risk of getting OA in the other knee and, unlike those who are not yet symptomatic, may be motivated to adopt preventive strategies. Thus, a focus on those with painful knee $\mathrm{OA}$ in at least $1 \mathrm{knee}$ is a natural strategy for prevention.

Prevention is usually conceptualized as primary, secondary, or tertiary, the latter connoting treatment of existing disease ${ }^{5}$. We shall contend that secondary prevention (after initial symptoms with a goal to prevent recurrence or progression of those symptoms) is an appealing option for knee OA. The goals of secondary prevention would differ depending on the state of the contra-

Personal non-commercial use only. The Journal of Rheumatology Copyright $\odot$ 2013. All rights reserved. 
lateral knee. For those who have OA in only 1 knee, goals would include prevention of pain or disease in the other knee, and for persons in whom the other knee is already affected, the goal would be to prevent disease progression in that other knee as well as the originally affected knee.

In individuals with OA in 1 knee, the contralateral knee is at a high risk of concurrent or later $\mathrm{OA}^{6,7,8}$. This raises the question of how disease occurrence or progression might be prevented. If knee OA occurs more often in the medial compartment, and if bilateral, in the same compartment in both knees, then it is possible that reducing load in the medial compartment of both knees could alleviate pathological loading, first in the already affected knee and then in the contralateral knee. However, to our knowledge, there are no data on the extent to which medial disease is bilateral (only that OA is often bilateral).

The external knee adduction moment (EKAM) is generally used as a surrogate measure of medial loading. During walking, the ground reaction force passes medial to the knee in the frontal plane, creating a moment that adducts the tibia relative to the femur. In healthy gait the peak force on this compartment is almost 2.5 times more than that on the lateral compartment ${ }^{9}$. In persons with medial knee OA, the EKAM has been shown to correlate with severity ${ }^{10}$, progression of disease ${ }^{11}$, and reduction in cartilage thickness $^{12}$. In individuals without knee pain, higher EKAM have been associated with the risk of developing knee $\mathrm{OA}^{13}$.

Because focal mechanical loads play a role in the development and progression of knee OA, load-modifying interventions are particularly appealing because of the low risk and low costs associated with this treatment method compared to some pharmacological and surgical interventions.

Many methods exist that can lower medial load and could be attempted in prevention strategies. One such method is the use of lateral wedge insoles ${ }^{14}$. Lateral wedge insoles are placed inside shoes and are intended to reduce the moment arm of the ground reaction force vector relative to the knee joint center during walking ${ }^{15}$. Many researchers have shown that lateral wedges reduce the peak EKAM by about $5 \%$ on the affected side ${ }^{15,16,17,18}$, although they do not necessarily reduce knee pain ${ }^{19,20}$. All studies to date have addressed the EKAM of affected knees in patients with medial compartment knee OA. This is partly because wedge insole trials do not usually include treatment of the contralateral knee, which is often treated with a neutral shoe insert ${ }^{19}$. As data are available on longterm followup of individuals and the subsequent risk of contralateral OA, information on the changes from lateral wedge insoles on the less-affected side are needed.

This study hypothesized first that medial knee OA was often symmetric either concurrently or that there was a high risk of later development of medial knee OA on the contralateral side. We also hypothesized that in addition to decreasing medial loading of the more affected knee, lateral wedge insoles would reduce medial loading on the contralateral side. To address the first hypothesis, we used data from the Framingham Osteoarthritis Study to determine the proportion of those with medial knee OA who might be at risk of either concurrent or future contralateral medial knee OA. To address the second hypothesis, we used data from an ongoing study of lateral wedge insoles in which we used these insoles in both shoes in persons who had painful medial knee OA on at least 1 side.

\section{MATERIALS AND METHODS}

Our first question was how often persons with medial OA on 1 side had either concurrent or later medial OA on the contralateral side. Because a short-term trial is not optimal to address this question and does not provide generalizable estimates, we turned to data from the Framingham Study to address this issue. Second, we wished to know whether an intervention known to reduce medial loading in knees with medial OA might reduce medial loading in the contralateral knee. Institutional Review Board and UK National Health Services Research Ethics approval was obtained for both studies.

Frequency of bilateral medial $O A$. We used the Framingham data ${ }^{21}$ to determine the proportion of contralateral knees that might benefit from a reduction in the EKAM. That large community-based study included weight-bearing anteroposterior knee radiographs obtained from subjects at baseline and, on average, $8.6 \pm 1.1(\mathrm{SD})$ years later (range 6.9-11.7). The baseline data allowed us to determine the prevalence of bilateral medial OA and the longitudinal data to examine, in the knees with unilateral medial knee OA at baseline but with no OA on the contralateral side, what proportion of knees developed medial OA on that side.

Of the different cohorts studied in Framingham, we focused on the Heart Study Offspring who had followup radiographs and whose evaluation (1992-1995 baseline and 2002-2005 followup) was more recent than the original Heart Study cohort. All subjects had standing anteroposterior knee radiographs and were also asked whether they had knee pain, aching, or stiffness on most days. On the radiograph, medial knee OA was defined as above Kellgren and Lawrence grade $\geq 2$ with medial greater than lateral joint space narrowing (JSN; measured on a 0-3 scale). We also defined symptomatic medial OA as present when a subject had both medial radiograph knee OA (as above) and responded "yes" to the question on knee pain, indicating the knee in which medial knee OA was present. We focused on those with longitudinal followup data and identified those who had at least 1 knee with medial OA and no knee replacements. Details of radiograph acquisition and reading protocols and reader reliability have been described elsewhere ${ }^{21}$. We carried out 2 analyses, 1 of those with medial radiograph $\mathrm{OA}$ and the other of those with symptomatic medial knee OA.

Use of a lateral wedge insole to lower the adduction moment in the contralateral knee. Participants with knee pain were recruited from orthopedic clinics, physiotherapy clinics, and advertisements in local media for a trial testing shoe inserts and wedges. The eligibility criteria for participation in the study were aged 45 years and above, with medial tibiofemoral OA with Kellgren and Lawrence grade 2 or 3 on radiograph of the affected painful knee with medial greater than lateral JSN, and at least mild pain when walking on a flat surface during the last week, assessed by the Knee Injury and OA Outcome Score pain subscale (P5) ${ }^{22}$. Radiographs were generally acquired as part of the patient's routine care and were read for the study by an experienced academically based musculoskeletal radiologist according to the Osteoarthritis Research Society International atlas ${ }^{23}$. Patients were excluded if they presented with pain more localized to the patellofemoral joint on examination than to the medial joint (wedge inserts are not appropriate for disease in the patellofemoral compartment, and lowering the EKAM may make it worse), had tricompartmental knee OA, or grade 1 or grade 4 tibiofemoral $\mathrm{OA}$ on the Kellgren and Lawrence scale. Other

Personal non-commercial use only. The Journal of Rheumatology Copyright $\subset$ 2013. All rights reserved. 
reasons for exclusion included a history of high tibial osteotomy or other realignment surgery, total knee replacement on the affected side, any foot and ankle problems (such as hallux valgus, plantar fasciitis, or peripheral neuropathy), or any foot and ankle pain that contraindicated the use of load-modifying footwear interventions. In addition, participants were excluded if they had severe coexisting medical morbidities or used orthoses prescribed by a podiatrist or orthotist. Eligible participants were invited to attend the gait laboratory, where informed consent was obtained.

Interventions. We conducted a single-visit randomized trial testing different wedges and shoes for their effect on the EKAM. Two of these interventions were lateral wedges that have been shown in prior studies to reduce EKAM in patients with medial knee $\mathrm{OA}^{18,24}$. Both lateral wedge insoles consisted of a $5^{\circ}$ wedge that was posted just proximal to the fifth metatarsal head to ensure fitting in the toe box of the shoe. The wedges were used on both the affected and contralateral limbs of all participants (i.e., they were applied bilaterally). The major difference between the lateral wedge insoles is that 1 had a medial support ${ }^{24}$, which was expected to be more comfortable, whereas the other lateral wedge had no support ${ }^{18}$ (Figure 1). Previous research ${ }^{25}$ has shown that individuals have differing responses to the different lateral wedges and therefore both were assessed to determine the effectiveness of each. During the trial, these lateral wedges were inserted into a flat-soled control shoe (Ecco Zen), with subjects having a minimum 5-minute familiarization period (Figure 1).

Protocol. All participants underwent baseline gait analysis in the control shoes and while wearing both types of lateral wedge insoles with a reference trial collected for each condition. The order of presentation of the different conditions was randomized prior to participants' enrollment, using computer-generated permutations. A 16-camera Qualisys OQUS3 motion analysis system operating at $100 \mathrm{~Hz}$ and 4 AMTI BP400600 force plates operating at $200 \mathrm{~Hz}$ were used to measure the kinematics and kinetics during the trials. Each subject completed a minimum of 3 successful trials at a self-selected walking speed. A trial was defined as successful when force platform contacts were made so that comparison between sides could be accomplished. The CAST marker set technique ${ }^{26}$ was used, whereby rigid clusters of 4 nonorthogonal markers were positioned over the lateral shank, lateral thigh, and sacrum to track the movements of the limbs. Retroreflective markers were glued securely to the control shoes with the foot modeled as a rigid segment. A reference trial was collected in which retroreflective markers were placed on bony landmarks to specify the location of these in relation to the clusters and to approximate the joint center. Ankle and knee joint centers were calculated as midpoints between the malleoli and femoral epicondyles, respectively. The hip joint center was calculated using the regression model of Bell, et $a l^{27}$, based on the anterior and posterior superior iliac spine markers. Using an inverse dynamic approach, Visual 3D (C-Motion), we calculated the EKAM during stance phase for all of the trials and conditions. A custom Matlab program was used to extract the maximum EKAM during early stance (up to 50\% of stance phase) and to calculate the knee adduction angular impulse $(\mathrm{KAAI})^{28}$, which is the area under the adduction moment curve during the entire stance phase of gait. Because individuals with knee OA have an increased duration of stance, the KAAI was seen as an appropriate addition to the EKAM; KAAI gives a measure of loading over the stance phase and not at 1 particular point. EKAM were normalized to subject mass $(\mathrm{Nm} / \mathrm{kg})$, with the KAAI normalized to subject mass and time $\left(\mathrm{Nm} / \mathrm{kg}^{*} \mathrm{~s}\right)$.

Data analysis. Because each of the lateral wedge insoles was designed to reduce EKAM and KAAI, and because we found similar results for them, we took the mean reduction in EKAM and KAAI for the 2 insoles and used this mean as our measure of EKAM and KAAI during use of lateral wedges. It was thought that this would produce a more general picture of wedge use than would data on 1 specific wedge alone. Our main focus was to determine whether the EKAM and KAAI in the contralateral limb were reduced during use of the lateral wedge versus the control shoe. For each person, we calculated this as follows for the contralateral limb:

$$
\frac{\text { (Mean EKAM when wearing a wedge }- \text { EKAM in control shoe) }}{\text { EKAM in control condition }} \times
$$

This expresses change in EKAM as a percentage of the control condition. Change in KAAI was calculated using the same method.

As a secondary analysis, we compared the 2 limbs regarding wedge-induced changes in EKAM and KAAI. Because the distribution of percentage changes was skewed, nonparametric confidence intervals were calculated (using the binomial method) around the percentage changes, to assess the significance of the change. Statistical analysis was performed using Stata Version 11.2 (StataCorp) with the significance level set at $\mathrm{p}<$ 0.05 (where significance tests were used).

\section{RESULTS}

Of 1278 Framingham Offspring subjects who had longitudinal radiographs, $185(14.5 \%)$ had at least 1 knee with radiographic OA (Kellgren and Lawrence grade $>2$ ) at baseline. Of these, 23 had only lateral OA (where lateral JSN was greater than medial JSN), 9 had equal scores for

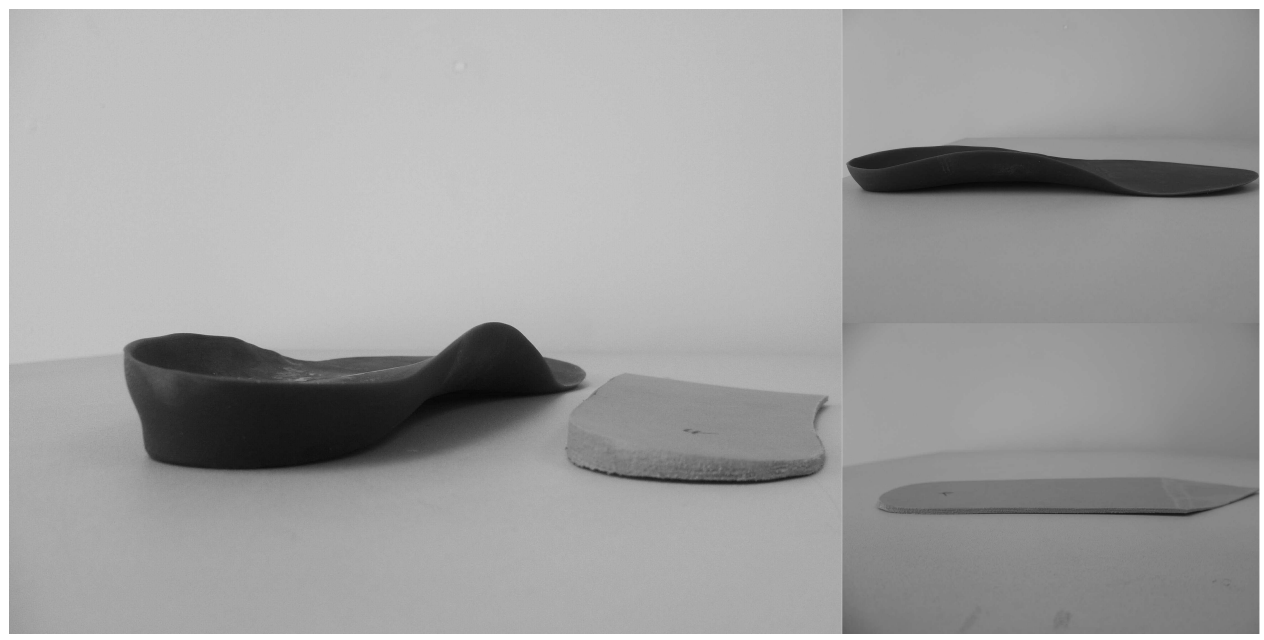

Figure 1. The 2 different lateral wedge insoles used in the study, showing the increased medial support in one insole. 
lateral and medial JSN, and 1 had a contralateral knee replacement. The 152 remaining subjects had medial OA in at least 1 knee and a contralateral native knee. The mean age of these subjects was 58.7 years (SD 8.4), mean body mass index (BMI) was 29.7 (SD 5.7), and there were 74 men (48.7\%) and 78 women (51.3\%). Forty-three percent of these knees were painful on most days and in 32 (21.3\%), there was a recalled major knee injury. Of these subjects with OA predominantly in the medial compartment in 1 knee, 84/152 (55.3\%) had OA bilaterally (Table 1). Of these 84 persons, 79 (94\%) had bilateral medial OA. Of the 152, 68 had no radiograph OA at baseline on the contralateral side. At followup, 60/68 (88.2\%) had developed OA on the contralateral side and of these, almost all $(58 / 60 ; 96.7 \%)$ had developed medial disease. Thus, of the original 152 subjects with medial OA, 137 (90\%) either had or later developed medial OA in the contralateral knee.

Fewer subjects had medial symptomatic knee OA ( $\mathrm{n}=$ 67) at baseline. Of these, 29 (43\%) had medial symptomatic knee OA on the contralateral side. Of the 38 knees without symptomatic knee OA at baseline who were restudied, 14 (37\%) developed medial symptomatic knee OA. Thus, of the 67 with medial symptomatic knee OA, 43 (64\%) had or developed medial symptomatic knee OA on the contralateral side.

We studied 51 participants ( 29 male and 22 female) with radiographically confirmed painful medial knee OA. Mean age was 59.6 years (SD 8.9), mean height $1.69 \mathrm{~m}$ (SD 0.08), mean weight $90.3 \mathrm{~kg}$ (SD 17.9), and mean BMI 31.6 (SD 5.07). Twenty-two demonstrated grade 2 (mild) disease on radiograph, with the remaining 29 demonstrating grade 3 (moderate) disease. Walking speed during the 3 conditions (control and 2 lateral wedge conditions) showed no statistical difference $[1.14 \mathrm{~m} / \mathrm{s}$ (SD 0.22); $1.15 \mathrm{~m} / \mathrm{s}$ (SD 0.23); and $1.15 \mathrm{~m} / \mathrm{s}$ (SD 0.22), respectively]. Twelve individuals had radiographs of both knees available. Of those, 6 had bilateral radiographic medial $\mathrm{OA}$ and 6 had unilateral medial OA. None had medial disease on 1 side and lateral on the other. All noted pain on the affected side but 33 of 51 $(65 \%)$ subjects reported no knee pain in the contralateral knee.

At baseline, neither EKAM nor KAAI in the control condition differed significantly between the affected and the contralateral knee (median difference in EKAM 0.03 $\mathrm{Nm} / \mathrm{kg}$; $95 \%$ CI -0.03 to 0.07 ; median difference in KAAI $0.00 \mathrm{Nm} / \mathrm{kg}$; $95 \% \mathrm{CI}-0.02$ to 0.03 ; Table 2).

For the affected knees, there was a significant reduction in EKAM (median change $-4.84 \%$; $95 \% \mathrm{CI}-11.33 \%$ to $-0.65 \%$ ) and in KAAI (median change $-6.38 \%$; $95 \%$ CI $-8.89 \%$ to $-2.64 \%$ ). For the contralateral limb, there were also significant reductions in both early stance EKAM (median change $-9.34 \%$; 95\% CI $-10.57 \%$ to $-6.45 \%$ ) and KAAI (median change $-11.45 \%$; $95 \%$ CI -14.81 to -6.52 ).

Comparing the affected and contralateral sides, the changes in EKAM and KAAI observed did not differ significantly between legs (median EKAM difference in change between legs $3.23 \%$; $95 \%$ CI $-2.52 \%$ to $7.72 \%$; median KAAI difference in change between legs $2.68 \%$; $95 \%$ CI $-1.08 \%$ to $8.65 \%)$.

\section{DISCUSSION}

Lateral wedges reduce medial loading in knees with medial OA. Our data suggest that the contralateral knee is also at high risk of either concurrent or future medial OA. We suggest that medial unloading effects of lateral shoe wedges occur not only in the affected knees but also in the contralateral knees. Notably, the reduction in medial loading in the contralateral limb may be as great as or greater than that in the affected limb. Our findings may have important implications for disease prevention.

While previous studies have suggested that in persons with unilateral knee OA, the contralateral knee is at high risk of disease, to our knowledge this is the first study to evaluate the symmetry of compartment-specific risk in knee $\mathrm{OA}$. We found a surprisingly high rate of symmetry for medial knee OA; among Framingham participants with medial knee OA, $90 \%$ either had or developed contralateral medial knee OA by radiograph, and when we looked at the smaller number with medial symptomatic OA, 64\% either had or developed it on the contralateral side. Because knee symptoms fluctuate, it is likely that persons who at the time of the baseline or followup Framingham examinations did not note knee symptoms had such symptoms at other junctures. Few persons with medial OA on 1 side (4.6\%) either had or developed lateral disease; the ratio of medial/lateral OA in these subjects was $18: 1$. Only a few at

Table 1. The radiograph status of the contralateral knee in 152 Framingham subjects with medial knee osteoarthritis (OA), including cross-sectional and longitudinal results. Average followup $8.6 \pm 1.1$ (SD) years; range 6.9 to 11.7 years. Data are n $(\%)$.

\begin{tabular}{lccc}
\hline Status & $\begin{array}{c}\text { Without OA in } \\
\text { Contralateral Knee }\end{array}$ & $\begin{array}{c}\text { With Lateral OA in } \\
\text { Contralateral Knee }\end{array}$ & $\begin{array}{c}\text { With Medial OA in } \\
\text { Contralateral Knee }\end{array}$ \\
\hline $\begin{array}{c}\text { OA status at baseline } \\
\begin{array}{l}\text { OA status at followup (only in } \\
\text { those with no OA at baseline) }\end{array}\end{array}$ & $88(45)$ & $5(3)$ & $79(52)$ \\
OA status in all subjects at followup & $8(5)$ & $2(3)$ & $58(85)$ \\
\hline
\end{tabular}

Personal non-commercial use only. The Journal of Rheumatology Copyright @ $\odot 2013$. All rights reserved. 
Table 2. Knee loading measures in control (no wedge) and wedged (average of 2 lateral wedges) conditions, and associated percentage changes and differences.

\begin{tabular}{|c|c|c|c|}
\hline & $\begin{array}{c}\text { Value Without } \\
\text { Wedge Applied } \\
\text { (control; median, IQR) }\end{array}$ & $\begin{array}{c}\text { Wedge Average } \\
\text { Value (experimental; } \\
\text { median, IQR) }\end{array}$ & $\begin{array}{c}\text { Percentage Change } \\
(95 \% \mathrm{CI})\end{array}$ \\
\hline \multirow[t]{2}{*}{ EKAM $(\mathrm{Nm} / \mathrm{kg})$ Affected leg } & 0.38 & 0.35 & -4.84 \\
\hline & 0.30 to 0.45 & 0.28 to 0.43 & $(-11.33$ to -0.65$)$ \\
\hline \multirow[t]{2}{*}{ Unaffected leg } & 0.34 & 0.32 & -9.34 \\
\hline & 0.23 to 0.45 & 0.21 to 0.40 & $(-10.57$ to -6.45$)$ \\
\hline \multirow[t]{2}{*}{ KAAI $\left(\mathrm{Nm} / \mathrm{kg}^{*} \mathrm{~s}\right)$ Affected leg } & 0.15 & 0.13 & -6.38 \\
\hline & 0.10 to 0.18 & 0.10 to 0.17 & $(-8.89$ to -2.64$)$ \\
\hline \multirow[t]{2}{*}{ Unaffected leg } & 0.14 & 0.13 & -11.45 \\
\hline & 0.10 to 0.18 & 0.08 to 0.17 & $(-14.81$ to -6.52$)$ \\
\hline
\end{tabular}

IQR: interquartile range; EKAM: external knee adduction moment, normalized to subject mass; KAAI: knee adduction angular impulse, normalized to subject mass and time.

the 9.5-year followup did not develop radiograph OA in the contralateral knee.

Increased EKAM have been linked with worsening of OA in the medial compartment ${ }^{11,29}$. High EKAM also predict the development of new knee pain in persons without $i^{13}$. Shakoor, et $a l^{30}$ recently reported that, in those undergoing unilateral hip replacement, the high risk of contralateral knee $\mathrm{OA}$ is preceded by a high EKAM and increased medial bone density. Thus, an increased EKAM may lead to further development and progression of medial knee OA. Walter, et $a l^{31}$ proposed that a decreased EKAM does not necessarily reduce medial contact force, primarily because of an increase in the knee flexion moment. In our study there was no difference in the peak knee flexion moment between the intervention and control and therefore, in agreement with previous findings ${ }^{32}$, a reduction in the peak EKAM on the affected and contralateral side, in the absence of changes in the level of muscle co-contraction, is likely to correspond with a decrease in the medial contact force.

Our study has shown that lateral wedge insoles substantially reduce the EKAM and KAAI on the contralateral limb. As shown also in other studies, lateral wedge insoles reduce the peak EKAM by about $5 \%$ on the affected side. The reduction in contralateral loading that we report has the potential to reduce OA development and progression in the other knee. Because EKAM is determined in part by the location of the center of mass, which is a consequence of trunk lean, the effect of shoe wedges on the contralateral limb may have differed empirically from its effect on the ipsilateral limb. One potential reason for the large reductions in EKAM on the contralateral limb is that the disease process was milder on this side. This is suggested in our sample by the absence of contralateral knee pain in most subjects. Investigators have reported that heel wedge response is greater when disease is mild ${ }^{33}$. It is important to note, however, that compared with control insoles, lateral wedge insoles do not reduce knee pain according to the results of at least 2 randomized clinical trials ${ }^{19,20}$.
One of the great challenges in preventing $\mathrm{OA}$ is that persons with early disease may not have sufficient symptoms to be motivated to adopt a preventive intervention. One way around that problem is to consider preventive interventions when a person has OA in 1 painful knee and is at high risk to get disease in the other knee. Data from the Framingham Study suggest that a remarkably high percentage of persons with medial knee OA either already has contralateral medial OA or is at high risk of getting disease in the medial compartment. Such subjects would be good targets for disease prevention. Given their symptoms, many patients might be motivated to adopt a disease prevention strategy. For OA prevention, this may constitute a "teachable moment" 34 , a health event that might motivate a person to adopt risk-reducing behavior. Teachable moments have been successfully used to encourage smoking cessation $^{34,35}$.

One limitation of our study was its short-term design; it would be encouraging if results were replicated in a longer-term study. However, previous research into the longevity of the effects of lateral wedge insoles ${ }^{17}$ demonstrated that they have similar biomechanical changes 1 month after using them. The choice of control shoe in the study could be criticized as this was a flat-soled shoe with no standard insole. However, the control shoe was chosen to mitigate any masking of the effects of the lateral wedge insoles. It is likely that different results would be seen with different control shoes. Further research is needed on the effect of shoes alone on lateral wedge effectiveness. The persons in our study had more symptomatic affected knees and in most, the contralateral knee was asymptomatic. We did not assess symptom response on the contralateral side in individuals, and this should be considered in future studies.

While we did not know the OA status of the contralateral knee in most cases, an analysis of Framingham data showed that the great majority of those with medial knee OA on 1 side have it on the other side or are at high risk of getting it there. Of those whose status we did know, all those with 
contralateral OA had medial OA. The similar EKAM in the contralateral knee to the affected knee may suggest medial disease there. Any future study of contralateral effects needs to include more comprehensive evaluations of this contralateral knee, and if prevention is intended, bilateral radiographs should be acquired and those with contralateral lateral disease removed.

Persons with medial knee OA in 1 knee are at high risk of having the same disease bilaterally either concurrently or in the future. Conservative management options such as lateral wedge insoles might prevent knee OA on the contralateral side. Future studies should identify the longer-term effects of lateral wedge insoles on the contralateral knee.

\section{ACKNOWLEDGMENT}

We acknowledge the valuable help of the Research into Osteoarthritis in Manchester research team for aiding with the recruitment and screening of the individuals, Manchester Academic Health Science Centre, and the National Institute for Health Research. We also appreciate the assistance of Jingbo Niu, DSc, at Boston University for analysis of the Framingham data, and Laura Heathers for technical assistance.

\section{REFERENCES}

1. Felson DT, Zhang Y. An update on the epidemiology of knee and hip osteoarthritis with a view to prevention. Arthritis Rheum 1998;41:1343-55.

2. Ahlback S. Osteoarthrosis of the knee. A radiographic investigation. Acta Radiol Diagn 1968;Suppl 277:7-72.

3. Felson DT, Nevitt MC, Zhang Y, Aliabadi P, Baumer B, Gale D, et al. High prevalence of lateral knee osteoarthritis in Beijing Chinese compared with Framingham Caucasian subjects. Arthritis Rheum 2002;46:1217-22.

4. Prodromos CC, Andriacchi TP, Galante JO. A relationship between gait and clinical changes following high tibial osteotomy. J Bone Joint Surg Am 1985;67:1188-94.

5. Gordon R. An operational classification of disease prevention. In: Steinberg JA, Silverman MM, editors. Preventing mental disorders: A research perspective. Rockville, MD: US Department of Health and Human Services; 1987.

6. Mont MA, Mitzner DL, Jones LC, Hungerford DS. History of the contralateral knee after primary knee arthroplasty for osteoarthritis. Clin Orthop Relat Res 1995;321:145-50.

7. Spector TD, Hart DJ, Doyle DV. Incidence and progression of osteoarthritis in women with unilateral knee disease in the general population: The effect of obesity. Ann Rheum Dis 1994;53:565-8.

8. Felson DT, Zhang Y, Hannan MT, Naimark A, Weissman B, Aliabadi $\mathrm{P}$, et al. The incidence and natural history of knee osteoarthritis in the elderly: The Framingham Osteoarthritis Study. Arthritis Rheum 1995;38:1500-5.

9. Schipplein OD, Andriacchi TP. Interaction between active and passive knee stabilizers during level walking. J Orthop Res 1991;9:113-9.

10. Sharma L, Hurwitz DE, Thonar EJ, Sum JA, Lenz ME, Dunlop DD, et al. Knee adduction moment, serum hyaluronan level, and disease severity in medial tibiofemoral osteoarthritis. Arthritis Rheum 1998;41:1233-40

11. Miyazaki T, Wada M, Kawahara H, Sato M, Baba H, Shimada S. Dynamic load at baseline can predict radiographic disease progression in medial compartment knee osteoarthritis. Ann Rheum Dis 2002;61:617-22.
12. Erhart J, Favre J, Andriacchi T. Walking loading at the knee predicts MRI-derived cartilage thickness changes in medial compartment knee osteoarthritis. Osteoarthritis Cartilage 2011;19:S45.

13. Amin S, Luepongsak N, McGibbon CA, LaValley MP, Krebs DE, Felson DT. Knee adduction moment and development of chronic knee pain in elders. Arthritis Rheum 2004;51:371-6.

14. Sasaki T, Yasuda K. Clinical evaluation of the treatment of osteoarthritic knees using a newly designed wedged insole. Clin Orthop Relat Res 1987;221:181-7.

15. Kakihana W, Akai M, Nakazawa K, Takashima T, Naito K, Torii S Effects of laterally wedged insoles on knee and subtalar joint moments. Arch Phys Med Rehabil 2005;86:1465-71.

16. Butler RJ, Marchesi S, Royer T, Davis IS. The effect of a subject-specific amount of lateral wedge on knee mechanics in patients with medial knee osteoarthritis. J Orthop Res 2007;25:1121-7.

17. Hinman RS, Payne C, Metcalf BR, Wrigley TV, Bennell KL. Lateral wedges in knee osteoarthritis: What are their immediate clinical and biomechanical effects and can these predict a three-month clinical outcome? Arthritis Rheum 2008;59:408-15.

18. Kerrigan DC, Lelas JL, Goggins J, Merriman GJ, Kaplan RJ, Felson DT. Effectiveness of a lateral-wedge insole on knee varus torque in patients with knee osteoarthritis. Arch Phys Med Rehabil 2002;83:889-93.

19. Baker K, Goggins J, Xie H, Szumowski K, LaValley M, Hunter DJ, et al. A randomized crossover trial of a wedged insole for treatment of knee osteoarthritis. Arthritis Rheum 2007;56:1198-203.

20. Bennell KL, Bowles KA, Payne C, Cicuttini F, Williamson E, Forbes A, et al. Lateral wedge insoles for medial knee osteoarthritis: 12 month randomised controlled trial. BMJ 2011;342:d2912.

21. Nguyen US, Zhang Y, Zhu Y, Niu J, Zhang B, Felson DT. Increasing prevalence of knee pain and symptomatic knee osteoarthritis: survey and cohort data. Ann Intern Med 2011; $155: 725-32$

22. Roos EM, Roos HP, Lohmander LS, Ekdahl C, Beynnon BD. Knee Injury and Osteoarthritis Outcome Score (KOOS) - Development of a self-administered outcome measure. J Orthop Sports Phys Ther 1998;28:88-96.

23. Altman RD, Gold GE. Atlas of individual radiographic features in osteoarthritis, revised. Osteoarthritis Cartilage 2007;15 Suppl:A1-56.

24. Jones RK, Nester CJ, Kim WY, Tyson S, Laxton P, Jari S, et al. Direct and indirect orthotic management of medial compartment osteoarthritis of the knee. Gait Posture 2006;24:S141-2.

25. Jones RK, Zhang M, Findlow AH, Liu AM, Laxton P. The biomechanical effects of a new off-the-shelf design of lateral wedge insole on the knee and ankle. Hum Mov Sci 2013; [in press].

26. Cappozzo A, Catani F, Croce UD, Leardini A. Position and orientation in space of bones during movement: Anatomical frame definition and determination. Clin Biomech 1995;10:171-8.

27. Bell AL, Brand RA, Pedersen DR. Prediction of hip joint centre location from external landmarks. Hum Move Sci 1989;8:3-16.

28. Thorp LE, Sumner DR, Block JA, Moisio KC, Shott S, Wimmer MA. Knee joint loading differs in individuals with mild compared with moderate medial knee osteoarthritis. Arthritis Rheum 2006;54:3842-9.

29. Mundermann A, Dyrby CO, Andriacchi TP. Secondary gait changes in patients with medial compartment knee osteoarthritis: Increased load at the ankle, knee, and hip during walking. Arthritis Rheum 2005;52:2835-44

30. Shakoor N, Block JA, Shott S, Case JP. Nonrandom evolution of end-stage osteoarthritis of the lower limbs. Arthritis Rheum 2002;46:3185-9. 
31. Walter JP, D’Lima DD, Colwell CW Jr, Fregly BJ. Decreased knee adduction moment does not guarantee decreased medial contact force during gait. J Orthop Res 2010;12:1548-53.

32. Zhao D, Banks SA, Mitchell KH, D'Lima DD, Colwell CW Jr, Fregly BJ. Correlation between the knee adduction torque and medial contact force for a variety of gait patterns. J Orthop Res 2007;25:789-97.

33. Keating EM, Faris PM, Ritter MA, Kane J. Use of lateral heel and sole wedges in the treatment of medial osteoarthritis of the knee. Orthop Rev 1993;22:921-4.
34. McBride CM, Emmons KM, Lipkus IM. Understanding the potential of teachable moments: The case of smoking cessation. Health Educ Res 2003;18:156-70.

35. Glasgow RE, Stevens VJ, Vogt TM, Mullooly JP, Lichtenstein E. Changes in smoking associated with hospitalization: quit rates, predictive variables, and intervention implications. Am J Health Promot 1991;6:24-9. 